University of Nebraska - Lincoln

DigitalCommons@University of Nebraska - Lincoln

August 2004

\title{
DNA Strand Breaks Induced by 0-4 eV Electrons: The Role of Shape Resonances
}

\author{
Frédéric Martin \\ Université de Sherbrooke \\ Paul Burrow \\ University of Nebraska-Lincoln, pburrow1@unl.edu \\ Zhongli Cai \\ Universite' de Sherbrooke \\ Pierre Cloutier \\ Universite' de Sherbrooke \\ Darel Hunting \\ Universite' de Sherbrooke, \\ See next page for additional authors
}

Follow this and additional works at: https://digitalcommons.unl.edu/physicsburrow

Part of the Physics Commons

Martin, Frédéric; Burrow, Paul; Cai, Zhongli; Cloutier, Pierre; Hunting, Darel; and Sanche, Léon , "DNA Strand Breaks Induced by 0-4 eV Electrons: The Role of Shape Resonances" (2004). Paul Burrow Publications. 2. https://digitalcommons.unl.edu/physicsburrow/2

This Article is brought to you for free and open access by the Research Papers in Physics and Astronomy at DigitalCommons@University of Nebraska - Lincoln. It has been accepted for inclusion in Paul Burrow Publications by an authorized administrator of DigitalCommons@University of Nebraska - Lincoln. 
Authors

Frédéric Martin, Paul Burrow, Zhongli Cai, Pierre Cloutier, Darel Hunting, and Léon Sanche 


\title{
DNA Strand Breaks Induced by 0-4 eV Electrons: The Role of Shape Resonances
}

\author{
Frédéric Martin, ${ }^{1}$ Paul D. Burrow, ${ }^{2}$ Zhongli Cai, ${ }^{1}$ Pierre Cloutier, ${ }^{1}$ Darel Hunting, ${ }^{1}$ and Léon Sanche ${ }^{1, *}$ \\ ${ }^{1}$ Faculty of Medicine, Université de Sherbrooke, Quebec, J1H 5N4, Canada \\ ${ }^{2}$ Department of Physics and Astronomy, University of Nebraska-Lincoln, Lincoln, Nebraska 68588-0111, USA
}

(Received 15 March 2004; published 3 August 2004)

\begin{abstract}
Collisions of $0-4 \mathrm{eV}$ electrons with thin DNA films are shown to produce single strand breaks. The yield is sharply structured as a function of electron energy and indicates the involvement of $\pi^{*}$ shape resonances in the bond breaking process. The cross sections are comparable in magnitude to those observed in other compounds in the gas phase in which $\pi^{*}$ electrons are transferred through the molecule to break a remote bond. The results therefore support aspects of a theoretical study by Barrios et al. [J. Phys. B 106, 7991 (2002)] indicating that such a mechanism could produce strand breaks in DNA.
\end{abstract}

PACS numbers: $87.50 . \mathrm{Gi}, 34.50 . \mathrm{Gb}, 34.80 . \mathrm{Ht}, 87.14 . \mathrm{Gg}$

Introduction.-Many investigations during the last century have been devoted to the study of alterations induced by high-energy radiation in biological systems, more particularly within living cells and the DNA molecule. The biological effects of such radiation are usually not produced by the impact of the primary quanta, but rather by the secondary species generated along the radiation track [1]. As these species further react within irradiated cells, they can cause mutagenic, genotoxic, and other potentially lethal DNA lesions [2], such as singleand double-strand breaks (SSBs and DSBs).

Secondary electrons produced with energies below $20 \mathrm{eV}$ are the most abundant of the secondary species $[3,4]$, and their energy distribution is further degraded through ionization and inelastic collision processes. To understand the effects of radiation in cells, it is therefore crucial to determine the damage induced by such electrons on vital cellular components, particularly DNA. In experiments directly exposing DNA on a surface to an electron beam, Boudaiffa et al. [5] found that SSBs and DSBs could be produced at much lower energies than previously observed. The strand break yield as a function of electron impact energy peaked near the thresholds for electronic excitation, and the authors suggested that the process takes place through short-lived core-excited anion states, whose "parents" are the excited states of the neutral molecule [6]. Later Pan et al. [7] demonstrated that the dissociative electron attachment (DEA) process contributes significantly to these breaks. At still lower energies, electrons captured temporarily by simple molecules into normally empty valence orbitals (i.e., shape resonances) can also produce bond breaking through DEA, if the latter is exothermic [6]. Two properties of shape resonances are particularly relevant to the present work. Because the magnitude of the capture cross section for formation of a resonance varies inversely with the resonance energy, the cross sections may be very large if these resonances lie at low energy [6]. Furthermore, the lifetimes of temporary anion states increase as their energies decrease [6]. This combination of properties suggests that, if present, low-lying shape resonances in DNA could be highly efficient at breaking DNA strands. Recently, a number of theoretical and experimental investigations [8-11] on basic DNA constituents have generated considerable interest [12] in this possibility.

In the present Letter, we show that single strand breaks in DNA are produced at energies as low as the nominal zero energy threshold of the electron beam, and that the yield as a function of energy exhibits a sharp peak at $0.8 \pm 0.3 \mathrm{eV}$ and a broader feature centered at $2.2 \mathrm{eV}$. We compare these results to those obtained in gas-phase DEA $[10,11,13,14]$ and electron transmission spectroscopy (ETS) [15] experiments on the DNA bases and in theoretical studies $[8,9]$. We provide evidence that the SSBs are initiated in part by electron attachment into the empty $\pi^{*}$ valence molecular orbitals [16] of the DNA bases, and we show that the yield is comparable in magnitude to that observed in gas-phase DEA processes, in which electrons in $\pi^{*}$ orbitals are transferred to a "remote" bond that is subsequently broken. Direct attachment into low-lying $\sigma^{*}$ orbitals [16] of the phosphate group may also play a role, but gas-phase results supporting DEA via this process are less conclusive.

Procedure.-In the present experiment, supercoiled DNA [pGem-3Zf(-), 3197 base pairs] was prepared and purified as previously described [17]. An aqueous solution of the molecule as a sodium salt was obtained in the last step of the purification procedure, where the DNA was washed with a buffer containing sodium perchlorate. Thus, the negative charge of the phosphate groups is counterbalanced by $\mathrm{Na}^{+}$ions. Under a dry nitrogen atmosphere, $125 \mathrm{ng}$ of DNA [17] in $10 \mu \mathrm{l}$ of $\mathrm{H}_{2} \mathrm{O}$ was deposited on a chemically clean tantalum sheet, frozen at $-70^{\circ} \mathrm{C}$ and lyophilized with a hydrocarbon-free sorption pump at a pressure of 3 mTorr for two hours. Lyophilized DNA formed a film with a diameter of $3.5 \pm$ $0.2 \mathrm{~mm}$ that was directly transferred to an ultrahigh vacuum chamber (UHV). After a $24 \mathrm{~h}$ evacuation, the 
DNA was exposed to an electron beam at a background pressure of $5 \times 10^{-9}$ Torr at room temperature, at a fixed incident electron current of $2.0 \mathrm{nA}$ (current density of $1.6 \times 10^{10}$ electrons $\mathrm{s}^{-1} \mathrm{~cm}^{-2}$ ) and a constant incident electron energy, for irradiation times of seconds up to $4 \mathrm{~min}$. The energy resolution of the beam was $0.5 \mathrm{eV}$ fullwidth at half-maximum (FWHM). The beam energy was determined relative to the vacuum level within $\pm 0.3 \mathrm{eV}$ by measuring the energy for the onset of current transmitted through the film. The data was recorded in the energy range -0.1 to $4.1 \mathrm{eV}$ at $0.3 \mathrm{eV}$ intervals. The area of the electron beam was adjusted to be slightly smaller than that of the sample.

The SSBs and DSBs were detected by measuring the transformation induced by electron impact on DNA, from the initial supercoiled configuration of the molecule to the circular or linear forms. When a single strand is broken, the strained energy of the twisted supercoiled configuration is released causing the DNA topological configuration to become circular, whereas a cut of the two adjacent strands necessarily produces a linear DNA molecule. Once removed from the UHV chamber, the DNA was recovered and analyzed as previously described [17]. The different forms of DNA were separated by gel electrophoresis and the percentage of each form was quantified by fluorescence.

Exposure response curves were obtained for several incident electron energies. As an example, the inset of Fig. 1 shows the dependence of the percentage yields of circular DNA on irradiation time for $0.6 \mathrm{eV}$ electrons. The amount of the linear form of plasmid DNA was below the detection limit of $0.2 \mathrm{ng}$. Thus, induced DSBs are estimated to be less than one per $10^{5}$ electrons. During the first $20 \mathrm{sec}$, the percentage of circular DNA increases linearly with irradiation time, but saturates thereafter,

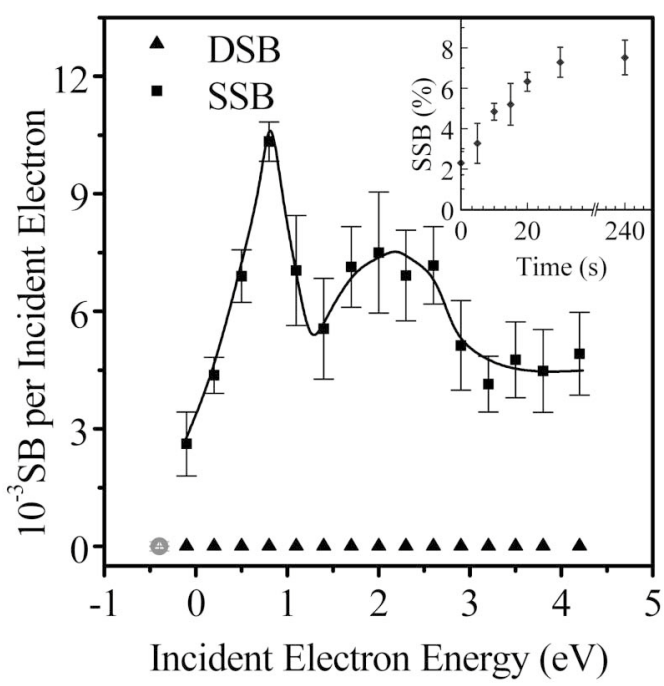

FIG. 1. Quantum yield of DNA single strand breaks (SSBs) and double-strand breaks (DSBs) vs incident electron energy. The inset shows the dependence of the percentage of circular DNA (i.e., SSBs) on irradiation time for $0.6 \mathrm{eV}$ electrons. possibly due to film charging, as seen from changes in the energy onset of electron current at the film substrate. Fluorescent microscopy shows that about $10 \%$ of the DNA film surface is covered with clusters, while the rest consists of a loosely packed monolayer of DNA. Charging is likely to arise from electron trapping within the clusters. Yields, e.g., DNA SSB or DSB per incident electron at each incident electron energy, were determined from the amounts of circular or linear DNA, respectively, resulting from $10 \mathrm{sec}$ of exposure (i.e., well within the initial linear regime of the exposure response curves) at the incident electron current and the known amount of DNA on the target. The loss of supercoiled DNA mirrors the formation of circular DNA. The samples were also placed under the electron gun for $10 \mathrm{sec}$ when a voltage of $-2.0 \mathrm{~V}$ was applied to the target, resulting in no current at the target. The absence of formation of circular and linear DNA in these controls confirms that heat and light from the electron gun do not cause any strand breaks.

Results and discussion.-Figure 1 shows the dependence of the yields of SSBs and DSBs on incident electron energy. In total, 80 different films were bombarded and analyzed to produce these results. The error bars show \pm the standard deviation from three to eight exposure experiments, each on separately prepared samples. Two peaks are observed in the yield function of SSBs at electron energies of 0.8 and $2.2 \mathrm{eV}$ with yields of $(1.0 \pm$ $0.1) \times 10^{-2},(7.5 \pm 1.5) \times 10^{-3}$ SSB per incident electron, respectively. The peaked structure in Fig. 1 provides unequivocal evidence for the role of low-lying temporary anion states in the bond breaking process. Of the basic molecular components comprising nucleic acids, only the resonances of the four DNA bases and the RNA base uracil have received significant attention over the energy range of interest. In the gas phase, sharp structures in the total scattering cross sections of the bases have been observed [15] over the range from 0.29 to $4.5 \mathrm{eV}$ using ETS and attributed to temporary electron occupation of the lowest $\pi^{*}$ valence orbitals. The assignment of these anion states, whose energies correspond to vertical attachment energies (VAE), is fully supported by Koopmans's theorem ab initio calculations, scaled to resonances in related molecules as described elsewhere [15].

The DEA process in the DNA bases has received much recent attention, and the yields of fragment anions in the gas-phase display significant resonance structure at low energies [10,13]. Scheer et al. [11] have analyzed the structures appearing in DEA and ETS studies of the bases and halo-substituted bases and attributed them to two mechanisms. The most prominent and narrow structures were assigned to vibrational Feshbach resonances [6] arising from mixing between the dipole bound states of the bases and the anion states associated with the lowest $\sigma^{*}$ valence orbitals. The remaining features were attributed to vibronic coupling between the valence $\pi^{*}$ and repulsive $\sigma^{*}$ anion states, giving rise to DEA peaks 
very near the energies of the $\pi^{*}$ resonances. This process has been well studied in unsaturated chlorocarbons [18]. Experimental results related to the empty orbitals of the remaining DNA components, the deoxyribose and phosphate groups, are sparse. A preliminary ETS study of trimethylphosphate [19], a surrogate for the DNA phosphate group, indicates a broad temporary anion state near $2 \mathrm{eV}$, consistent with calculations of the $\sigma^{*}$ lowest unoccupied molecular orbital (LUMO) anion state. Finally, the fully saturated deoxyribose group, consisting only of $\mathrm{O}, \mathrm{C}$, and $\mathrm{H}$ atoms, should also possess only short-lived $\sigma^{*}$ anion states.

A pronounced and narrow peak appears near $1 \mathrm{eV}$ in the DEA cross sections of uracil [10(a)], adenine, thymine [13], and thymidine [14] in the gas phase. Representative data for thymine [10(b)] are shown in the lower curve of Fig. 2, but shifted to lower energy by $0.2 \mathrm{eV}$ in order to match the location of the $0.8 \mathrm{eV}$ peak in the yield of SSBs. Such resonances might account for the $0.8 \mathrm{eV}$ peak in Fig. 1, if electron transfer to the backbone could occur from them and they exist in DNA. However, we note that the spacing of the two major peaks in the thymine DEA cross section does not match that of the strand breaks. The sharp $1 \mathrm{eV}$ peak in uracil has been assigned by Scheer $e t$ al. to a vibrational Feshbach resonance associated with a dipole bound anion state. Because the wave functions of such states are very diffuse, they overlap with other sites in the condensed phase and, in the absence of evidence to

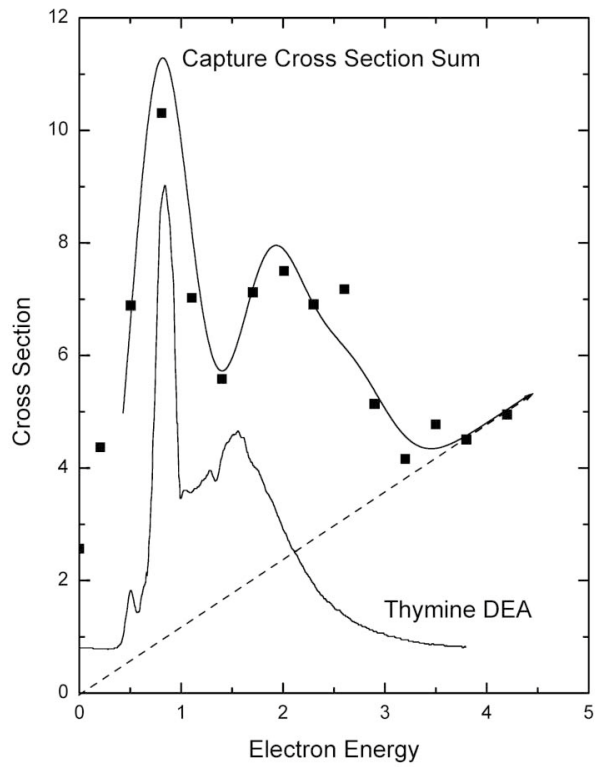

FIG. 2. Lower curve: the relative DEA cross section of thymine [10(b)], shifted by $0.2 \mathrm{eV}$ to lower energy. Upper curve: A model of the electron capture cross section of DNA as a function of electron energy based on the resonance energies of the bases and their widths determined in gas-phase scattering studies. The curve has been shifted to higher energy by $0.41 \mathrm{eV}$, normalized to the SSB data and a linearly increasing background (dashed line) added. Closed squares: SSB yields from Fig. 1. the contrary, we do not expect them to play a role in surface studies. On the other hand, the $\pi^{*}$ resonances associated with the LUMOs of thymine, cytosine, and guanine are sufficiently long lived in the gas phase to show clear evidence for anionic vibrational motion [15]. The natural width of the lowest, and largest, vibrational feature observed by ETS is $\leq 150 \mathrm{meV}$ in these compounds. This property thus argues for an assignment of the $0.8 \mathrm{eV}$ peak in Fig. 1 to a DEA process taking place through occupation of the LUMO $\pi^{*}$ orbitals of one or more bases.

Insight into the role of the $\pi^{*}$ anion states of the bases in bond breaking is found in a theoretical study by Barrios et al. [8]. In this work, a section of DNA was modeled that contains a cytosine base, a sugar ring, and the phosphate group. They find that an anionic potential surface exists that connects the initial $\pi^{*}$ anion state of the base to a $\sigma^{*}$ anion state. The latter leads to rupture of the $\mathrm{C}-\mathrm{O}$ bond connecting the phosphate group to the sugar. In other words, an electron placed on the base will migrate to the $\mathrm{C}-\mathrm{O} \sigma^{*}$ antibonding orbital as the latter bond length is stretched, leading to bond rupture. Transport of an electron from the base to the sugar-phosphate bond must take place through three saturated bonds. There is ample precedence for such transfers leading to bond breaking in gas-phase DEA studies. Pearl et al. [20,21] observed electron transfer from an ethylenic $\pi^{*}$ anion state to a C-Cl $\sigma^{*}$ anion state through two [20] and four [21] saturated bonds in two rigid chloronorbornene compounds. In each case, the maximum DEA cross section for production of $\mathrm{Cl}^{-}$occurs very near the VAE for formation of the $\pi^{*}$ resonance. The reaction in these compounds is exothermic because of the large electron affinity of the chlorine atom. It is instructive to compare the DEA cross sections in these compounds with the SSB yields found in the present work. In the chloronorbornene compound [20], the ratio of the DEA cross section at its $1.1 \mathrm{eV}$ peak to the theoretical maximum reaction cross section, $\pi \lambda^{2}$, is $5.4 \times 10^{-2}$, where $\lambda$ is the de Broglie wavelength of the electron at this energy. In the larger compound [21], in which transfer occurs through four saturated bonds, this ratio drops to $10^{-2}$. In the DNA base-sugar-phosphate system involving three saturated bonds, we would anticipate an intermediate value if the total DNA electron scattering cross section were entirely resonant in character. More reasonably, such a value should be considered an upper limit. As measured here, the SSB yield per electron of $10^{-2}$ in DNA at $0.8 \mathrm{eV}$ is entirely consistent with this picture. Considering the differences in the molecular systems compared above, this result should not be overinterpreted. However, it offers clear support for the charge transfer mechanism proposed by Barrios et al. [8].

To further support the role of the $\pi^{*}$ resonances, we simulate in an approximate manner the electron capture cross section as it might appear in DNA owing to the $\pi^{*}$ anion states of the bases. For this purpose, we represent 
each $\pi^{*}$ resonance by a Gaussian peak, for computational convenience, placed at each of the VAEs below $2.5 \mathrm{eV}$ determined by ETS [15]. The widths of the peaks, reflecting their natural lifetimes and Franck-Condon factors, correspond to the values observed by ETS. The peak magnitudes are scaled to reflect the inverse energy dependence of the electron capture cross sections, and the peaks are then convoluted with a Gaussian of FWHM of $0.5 \mathrm{eV}$ to represent the energy resolution of the present experiment. Under the assumption that equal numbers of each base are resident in our DNA, the contributions from each base are simply added. There is, of course, no assurance that the efficiencies for bond breaking will track the capture cross sections into the various resonances. These will depend on the lifetimes and relative energies of the $\pi^{*}$ and $\sigma^{*}$ anion states involved and the coupling between them. To facilitate comparison, the SSB data are reproduced in Fig. 2. The lowest peak in our modeled capture cross section, which occurs at $0.39 \mathrm{eV}$ in the gas phase, is shifted by $0.41 \mathrm{eV}$ to match that in the SSB yield and its magnitude normalized. A linearly increasing background, indicated by the dashed line, is added to the modeled cross section to compensate for the finite SSB yield just above the resonance region. The similarity between the modeled curve and the SSB data is quite striking. We note that the $0.41 \mathrm{eV}$ energy shift is only slightly outside the $\pm 0.3 \mathrm{eV}$ uncertainty in energy calibration. In moving from the gas phase to the condensed phase, we expect that polarization effects will shift the $\pi^{*}$ resonances observed in ETS to lower energies. However, the electric dipole fields created by the negatively charged phosphate groups and positive counter ions will likely play the major role. To the extent that the phosphate charge is closer to the bases, a net destabilization will occur. If our identification of the $0.8 \mathrm{eV}$ feature is correct, it appears that the destabilization owing to this effect balances or slightly exceeds that of the polarization induced by the transient anion. Returning to the role of $\sigma^{*}$ anion states, Li et al. [9] suggested theoretically that direct electron attachment into a dissociative anion state on the phosphate group could lead to $\mathrm{C}-\mathrm{O}$ bond rupture at the $3^{\prime}$ and $5^{\prime}$ positions of the sugar ring of the backbone, producing a SSB. Such a process may well contribute to the background on which the peaks in Fig. 1 are superimposed.

In conclusion, we have shown that electrons with energies well below the electronic excitation threshold can induce SSBs in DNA. Since these electrons are a major product of high-energy radiation $[3,4]$ and they have been shown to be involved in the mechanism of radiosensitization [22], the present results may have implications for enhancing the effectiveness of radiotherapy and for understanding the biological effects of low level radiation (e.g., at typical ground level of radiation each cell in our body sees on average seven low energy electrons per day [12]).
This work is supported by the Canadian Institutes of Health Research. We would like to thank Dr. Xifeng Li and Dr. Michael Huels for valuable discussions.

*Present address: Université de Sherbrooke, Département de medicine nucléaire et de radiobiologie, 3001, 12 Avenue Nord, Sherbrooke, Quebec, Canada J1H 5N4 Email address: Leon.Sanche@usherbrooke.ca

[1] C. von Sonntag, The Chemical Basis for Radiation Biology (Taylor and Francis, London, 1987).

[2] J. F. Ward, in Advances in Radiation Biology 5, edited by J.T. Lett and H. Adler (Academic Press, New York, 1977), pp. 181-239.

[3] J. A. LaVerne and S. M. Pimblott, Radiat. Res. 141, 208215 (1995).

[4] V. Cobut, Y. Frongillo, J. P. Patau, T. Goulet, M.-J. Fraser, and J.-P. Jay-Gerin, Radiat. Phys. Chem. 51, 229 (1998).

[5] B. Boudaiffa, P. Cloutier, D. Hunting, M. Huels, and L. Sanche, Science 287, 1658 (2000).

[6] H. Hotop, M.-W. Ruf, M. Allan, and I. I. Fabrikant, Adv. At. Mol. Opt. Phys. 49, 85 (2003).

[7] X. Pan, P. Cloutier, D. Hunting, and L. Sanche, Phys. Rev. Lett. 90, 208102 (2003).

[8] R. Barrios, P. Skurski, and J. Simons, J. Phys. Chem. B 106, 7991 (2002); J. Berdys, I. Anusiewicz, P. Skurski, and J. Simons, J. Am. Chem. Soc. (to be published).

[9] X. Li, M. D. Sevilla, and L. Sanche, J. Am. Chem. Soc. 125, 13668 (2003).

[10] (a) See, for example, G. Hanel , B. Gstir, S. Denifl, P. Scheier, M. Probst, B. Farizon, M. Farizon, E. Illenberger, and T. D. Maerk, Phys. Rev. Lett. 90, 188104 (2003); (b) S. Denifl, S. Ptasinska, M. Cingel, S. Matejcik, P. Scheier, and T. D. Maerk, Chem. Phys. Lett. 377, 74 (2003).

[11] A. M. Scheer, K. Aflatooni, G. A. Gallup, and P. D. Burrow, Phys. Rev. Lett. 92, 068102 (2004).

[12] See, for example, G. P. Collins, Sci. Am. 289, 26 (2003).

[13] H. Abdoul-Carime, S. Gohke, and E. Illenberger, Phys. Rev. Lett. 92, 168103 (2004).

[14] Abdoul-Carime, S. Gohlke, E. Fischbach, J. Scheike, and E. Illenberger, Chem. Phys. Lett. 387, 267 (2004).

[15] K. Aflatooni, G. A. Gallup, and P. D. Burrow, J. Phys. Chem. A 102, 6205 (1998).

[16] W. L. Jorgensen and L. Salem, The Organic Chemist's Book of Orbitals (Academic Press, New York, 1973).

[17] M. A. Huels, B. Boudaïfa, P. Cloutier, D. Hunting, and L. Sanche, J. Am. Chem. Soc. 125, 4467 (2003).

[18] See, for example, T. Skalicky, C. Chollet, N. Pasquier, and M. Allan, Phys. Chem. Chem. Phys. 4, 3583 (2002), and references therein.

[19] A. Modelli, P. D. Burrow, G. A. Gallup, and A. M. Scheer (to be published).

[20] D. M. Pearl, P. D. Burrow, J. J. Nash, H. Morrison, and K. D. Jordan, J. Am. Chem. Soc. 115, 9876 (1994).

[21] D. M. Pearl, P. D. Burrow, J. J. Nash, H. Morrison, D. Nachtigallova, and K. D. Jordan, J. Phys. Chem. 99, 12379 (1995).

[22] S. Cecchini, S. Girouard, M. A. Huels, L. Sanche, and D. Hunting, Radiat. Res. (to be published). 